\title{
Ulcerated Giant Galactocele in a 30-Year-Old Woman: A Case Report from the Tamale Teaching Hospital in the North Regional Capital - Ghana
}

\author{
Der Muonir Edmund ${ }^{1 *}$, Anwar Sadat Seidu², Sheriff Mohammed², Akorli Emmanuel ${ }^{3}$, Osman Imoro ${ }^{2}$ and \\ Martin Kyereh ${ }^{2}$
}

${ }^{1}$ Department of Pathology, School of Medicine and Health Science of the University for Development Studies and the Tamale Teaching Hospital, Ghana

${ }^{2}$ Department of General Surgery, School of Medicine and Health Science of the University for Development Studies and the Tamale Teaching Hospital, Ghana

${ }^{3}$ Department of Radiology, Tamale Teaching Hospital, Ghana

"Corresponding author: Edmund DE, Department of Pathology, School of Medicine and Health Science of the University for Development Studies and the Tamale Teaching Hospital, Ghana, Tel: +233208709807; E-mail: maadelle@yahoo.com

Received: December 01, 2020; Accepted: December 12, 2020; Published: December 20, 2020

\begin{abstract}
Galactocele is a non-neoplastic cystic breast lesion found commonly in postpartum or lactating women. It may mimic neoplastic lesion because of the timing of its occurrence and or the clinicopathological changes in the affected breast tissue. It may thus pose a diagnostic challenge to clinicians in the absence of advanced imaging technology and histopathological examination. We present a 30-year-old woman para $7^{(5 \text { alive, } 2 \text { dead })}$ from the northern region of Ghana with an ulcerated giant galactocele of 4 years duration. The lesion started after the fourth child (aged 4 years) and progressively increased over the years to the current size at presentation. A diagnosis of galactocele was made by the surgeon based on the physical examination findings, supported by breast ultrasonography. CT scan of the head did not find any brain lesion. Similarly, a serum prolactin level was moderately elevated. She had simple mastectomy and histopathological examination of the breast specimen reported the lesion as an ulcerated giant galactocele. Conclusion: In the absence of any detectable pituitary adenoma and markedly elevated serum prolactin level, we rely on the short pregnancy intervals, duct obstruction and the long-standing chronic inflammation reaction with the associated fibrosis as the aetiological factors responsible for the pathology seen in this current case report.
\end{abstract}

Keywords: Giant galactocele; Duct obstruction; Chronic inflammation; Northern Ghana

Citation: Edmund DM, Seidu AS, Mohammed S, et al. Ulcerated Giant Galactocele in a 30-Year-Old Woman: A Case Report from the Tamale Teaching Hospital in the North Regional Capital - Ghana. Clin Case Rep Open Access. 2020;3(4):176. 
www.yumedtext.com | December-2020 | ISSN: 2582-5038 | https://dx.doi.org/10.46527/2582-5038.176

\section{Introduction}

A galactocele is a rare form of an obstructive benign cystic lesion of the breast [1,2]. It mostly occurs in postpartum women, whether lactating or not [2,3]. This disease may mimic neoplastic and non-neoplastic diseases of the female breast because of timing of its occurrence [1,2,4-6]. Evidence from endocrinology and the histopathology; macroscopic and microscopic features suggest the pathogenesis of galactocele to revolve around; present or previous stimulation by prolactin, secretary breast epithelium, and obstruction and dilated breast ducts filled with milk produced in the surrounding breast [7,8]. Galactocele can occur in any age group, but commonly affects lactating women within 20-30-years [1-6,9,10].

Clinically, it may present as single mass or multiple masses (lactating women), unilateral or bilateral, non-tender, firm, discrete and freely movable. It may or may not be associated with a milky discharge from the nipple [5,9-13].

Galactocele as a benign breast lesion has been reported in previous studies in parts of Africa [12-16]. However, none of these studies documented a lesion of this large size with long standing history (neglected disease). We present a 30-year-old lactating woman from the northern region of Ghana with an ulcerated giant galactocele of more than 6 years duration. This case report illustrates how ignorance, overreliance on herbal medicine and delay in timely and appropriate decision taken can contribute to delay presentation at a health facility and the associated cost implication.

\section{Case Report}

A 30-year-old lactating woman, para $7^{(5 \text { alive, } 2 \text { dead })}$ from Tolon in the northern region of Ghana presented with left breast swelling of 6 years duration associated with pain and skin ulceration. The condition started as a small painless lump after her $4^{\text {th }}$ child (aged 4-years now). The last child is 1-year old; she had spontaneous abortions for the fifth and sixth pregnancies respectively (approximately one-year pregnancy intervals). The mass is associated with milky nipple discharge. She resorted to herbal medications (both oral and topical) with no improvement in the condition. She began to notice ulcerations on the inframammary surface of her left breast and so she decided to seek medical advice from the hospital on 16/02/19.

At the time of presentation, examination revealed a young woman in moderate pain, afebrile and not pale. The hydration was good. The left breast was massively enlarged $(35.0 \mathrm{~cm} \times 20.0 \mathrm{~cm})$, soft to cystic, with distended veins and ulceration on the undersurface of the breast (FIG. 1). There was no nipple change and the axillary lymph nodes were not enlarged. He had copious non-blood-stained milky discharge from the nipple.

Initial working diagnosis: Ulcerative left galactocele

\section{Investigations}

\subsection{Imaging}

a. Ultrasound: Breast ultrasound revealed dilated ducts in the left breast, average diameter of $3.08 \mathrm{~cm}$, filled with fluid. No focal mass or calcifications seen. Normal nipple areolar region. No axillary lymph nodes seen.

Conclusion: Features are suggestive of galactocele. 
b. Head CT scan: No lesion pituitary adenoma none any abnormality seen (FIG. 2).

Conclusion: Normal brain parenchyma.

\subsection{Full blood count:}

Essentially normal.

\subsection{Renal function test}

Normal

\subsection{Serum prolactin}

41.21ug/l (Normal range=3.34 ug/l - $26.72 \mathrm{ug} / \mathrm{l})$. She had simple mastectomy as a surgical management on 22/02/19.

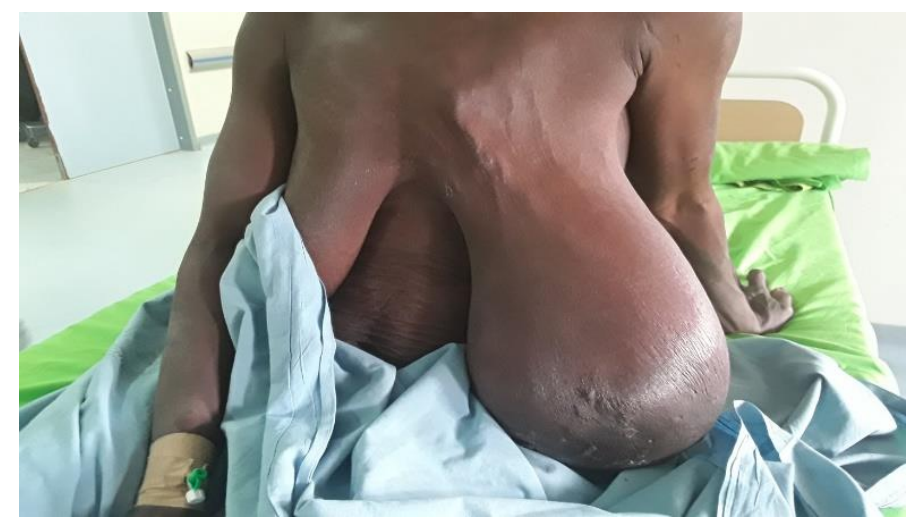

FIG. 1. A 30-year woman with massive soft to cystic left breast swelling associated with skin ulceration and distended blood vessels.

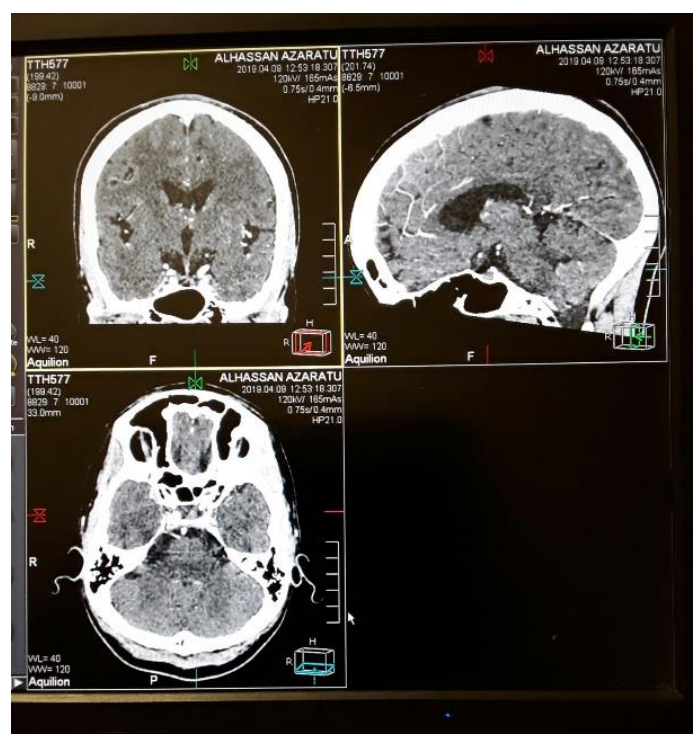

FIG. 2. CT scan of the brain of a 30-year-old lactating woman with a giant ulcerated galactocele; no lesion seen. 


\section{Histopathology}

Gross: Received a left simple mastectomy specimen $(20.5 \mathrm{~cm} \times 18 \mathrm{~cm} \times 12.0 \mathrm{~cm})$ with ulcerations on the skin. No axillary content. Slicing the breast revealed cysts of varying sizes filled with milk and pieces of fat that almost replaced the entire breast parenchyma. There were no calcifications seen, (FIG. 3).

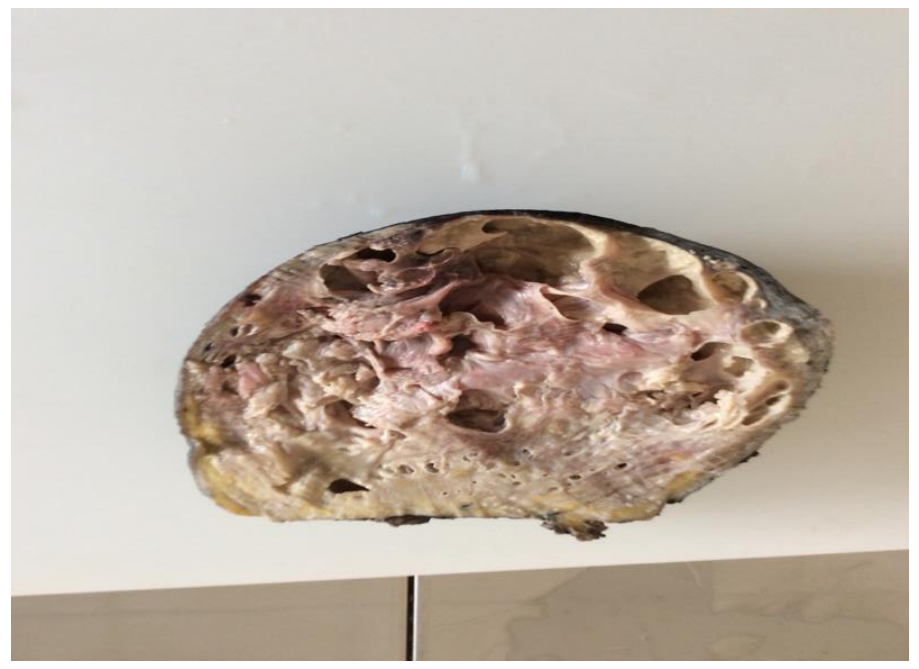

FIG. 3. Cut surface of a left simple mastectomy specimen from a 30-year-old lactating woman showing multiple cysts of varying sizes and shapes.

Microscopic: Sections from the breast show distended ducts with abundant secretory material and inflammatory cells in the lumen. The lining epithelium of the greatly dilated ducts is attenuated with periductal fibrosis. The less distended duct and acini are lined by secretory cells with hob-nail appearance vacuolated cytoplasm. There is marked stroma oedema, heavy mixed inflammatory cell infiltrates and necrosis. The myoepithelial cells are particularly attenuated and inconspicuous (FIG. 4).

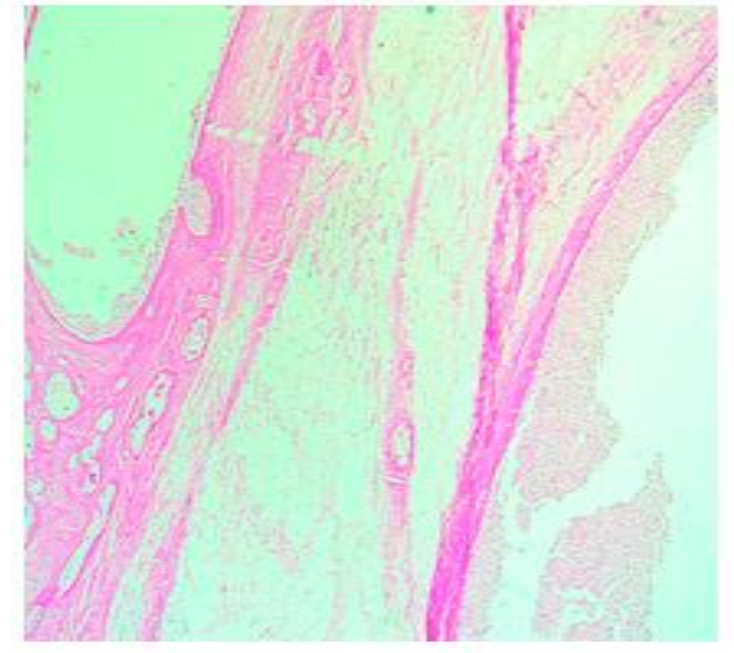

X4

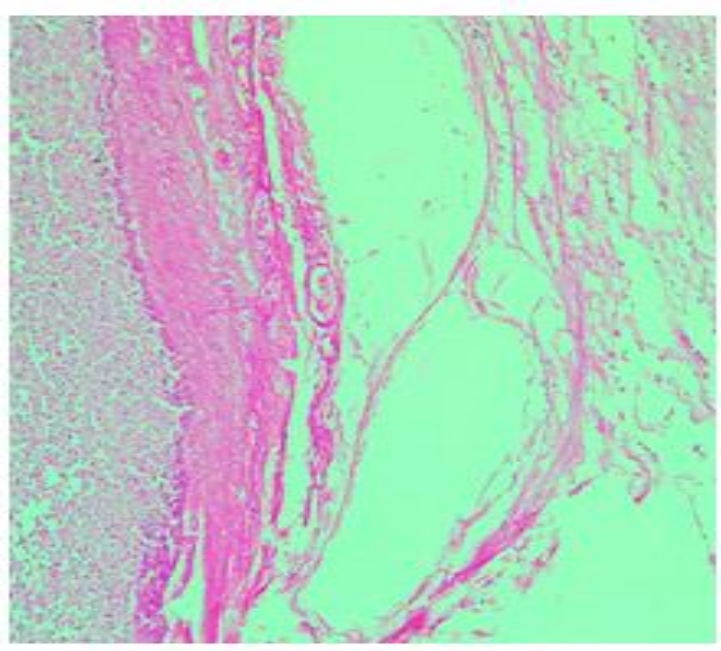

X10

FIG. 4. Histological section from the breast of a 30-year-old lactating woman at X4 and X10, showing dilated ducts filled milk, cellular debri and mixed inflammatory cell. 
www.yumedtext.com | December-2020 | ISSN: 2582-5038 | https://dx.doi.org/10.46527/2582-5038.176

\section{Discussion}

Galactocele is a non-neoplastic cystic breast lesion found commonly in postpartum or lactating women [1-3]. It may mimic neoplastic lesion because of the timing of its occurrence and or the physical features such as a palpable painless mas, skin changes and nipple abnormality [1,2,4-6]. It may thus pose a diagnostic challenge to clinicians in the absence of advanced imaging technology and histopathological examination. The current case report is that of a 30-year-old lactating woman, para $7^{(5 \text { alive, } 2 \text { dead) }}$ who presented with massive enlargement of the left breast of more than four years duration. The condition started after the fourth child (Aged 4-years) and gradually increased until the current state at presentation associated with skin ulcerations. Breast ultrasound examination found a lesion with features suggestive of a galactocele. The serum prolactin level was moderately elevated, $41.21 \mathrm{ug} / 1$ (Normal range=3.34 ug/l - $26.72 \mathrm{ug} / \mathrm{l}$ ), however, computerized tomography (CT) scan of the head with contrast did not reveal any pituitary adenoma, none any brain lesion. Histopathological examination of the mastectomy specimen submitted to Der Medical Diagnostic Centre Limited confirmed a galactocele. The woman's age at diagnosis and the fact that she was lactating at presentation clinically support previous studies that found galactocele to be a common pathological entity occurring in postpartum and or lactating women $[1-6,9,10]$.

The swelling started as a small single painless mass that progressively increased in size with each successive pregnancy and lactation. The clinical features of the current case report support previous studies that described galactocele as palpable painless mobile breast mass in a lactating woman [5,9-13]. However the uniqueness of this case report is reflected in the following parametres.

1. The progressive nature of the condition. The woman noticed the swelling after the $4^{\text {th }}$ child about 4 -years ago, and that the size kept increasing in size with successive pregnancies and lactation. Although the natural history of occurrence, the serum prolactin level and the pathological features of the disease in this current case report support the pathological basis of galactocele: present or previous stimulation by prolactin, secretory breast epithelium, obstructed and dilated breast ducts filled with milk, as in the published literature, $[7,8]$ the duration of the disease at presentation was too long and unacceptable. This may possibly be attributed to the painless nature of the disease and ignorance. Furthermore, the late state at presentation may result from the fact that more attention may have been directed at having more children, since she had two spontaneous abortions between the $4^{\text {th }}$ and the $7^{\text {th }}$ live births.

2. The huge size of the lesion in this case report. Large size galactoceles are commonly associated with pituitary adenoma $[15,17,18]$. The current case has no pituitary adenoma none any brain lesion. Again, large size galactoceles are associated with hyperprolactenaemia $[17,18]$. In the current case, serum prolactin was moderately elevated; $41.21 \mathrm{ug} / \mathrm{l}$ (normal range $=3.34 \mathrm{ug} / \mathrm{l}-26.72 \mathrm{ug} / \mathrm{l}$ ), and does not correlate with the long standing condition and the huge size of the lesion. In the absence of any detectable pituitary adenoma and markedly elevated serum prolactin level, $[17,18]$ we rely on the short pregnancy intervals (approximately 1-year), duct obstruction and the long-standing chronic inflammation reaction with the associated fibrosis as the aetiological factors responsible for the pathology seen in this current case report.

3. The long duration of the disease associated with skin ulcerations and pain as reported in this case report, may potentially suggest a neglected disease. In Ghana, and as in most parts of Africa, cultural taboos, influence of decision makers in a family regarding hospital attendance, the proliferation of herbal medicine, the myths about surgical intervention regarding breast lesion, cost and accessibility of health facilities have been identified as factors accounting for late presentation of patients to health facilities. Distance and accessibility to a health facility cannot be barriers that accounted for the delay in 
www.yumedtext.com | December-2020 | ISSN: 2582-5038 | https://dx.doi.org/10.46527/2582-5038.176

presentation in this case report. This is because this woman has a functional national health insurance card, lives in a district with a district hospital and also lives less than 15.0 kilometres from the Tamale Teaching Hospital. Likely factors operating in this case may be the influence of a decision maker, ignorance, and the over reliance on the herbal medicine. For instance, she admitted visits to herbalist and the use of herbal medicine in an attempt to treat the condition and that it was when all these steps taken did not achieve the expected outcome that she sought cure at the hospital. Again, at presentation and during the entire period of hospital admission, it was clear that the husband was the one taken all the decisions.

\section{Conclusion}

This case report illustrates how ignorance, overreliance on herbal medicine and delay in timely and appropriate decision taken can contribute to delay presentation at a health facility and the associated cost implication. In the absence of any detectable pituitary adenoma and markedly elevated serum prolactin level, we rely on the short pregnancy intervals, duct obstruction and the long-standing chronic inflammation reaction with the associated fibrosis as the aetiological factors responsible for the pathology seen in this current case report.

\section{Conflict of Interest}

There is no conflict of interest.

\section{Consent to Publish this Case Report}

We obtained consent from the relatives of the patient.

\section{Author's Contributions}

EDM drafted the case reports. SAS, SM OI, and MK provided the clinical history and performed the surgeries. KA provided the radiological images and the interpretations. EDM (Pathologist) prepared the tissue and reported the slides. EDM, SAS, SM, KA, OI and MK read through the case reports, edited and approved the final draft for publication.

\section{Acknowledgements}

We wish to express our profound gratitude to all the staff of Der Medical Diagnosis Centre Limited and the Department of General Surgery of the Tamale Teaching Hospital. We also would like to thank the patient and the husband for granting us permission to publish the data.

\section{REFERENCES}

1. Muttarak M, Padungchaichote W. Clinics in diagnostic imaging (84). Galactocoele. Singapore Med J. 2003;44(4):2115 .

2. Poiana C, Chirita C, Carsote M, et al. Galactocele and prolactinoma--a pathogenic association? Maturitas. 2009;62(1):98-102. 
www.yumedtext.com | December-2020 | ISSN: 2582-5038 | https://dx.doi.org/10.46527/2582-5038.176

3. Sawhney S, Petkovska L, Ramadan S, et al. Sonographic appearances of galactoceles. J Clin Ultrasound. 2002;30(1):18-22.

4. Kim MJ, Kim EK, Park SY, et al. Galactoceles mimicking suspicious solid masses on sonography. J Ultrasound Med. 2006;25(2):145-51.

5. Moukit M, Kouach J, El Hassani ME, et al. Galactocele mimicking a breast hydatid cyst in a pregnant woman. Saudi J Health Sci. 2017;6(1):62-4.

6. Vashi R, Hooley R, Butler R, et al. Breast imaging of the pregnant and lactating patient: Physiologic changes and common benign entities. AJR Am J Roentgenol. 2013;200(2):329-36.

7. Boyle M, Lakhoo K, Ramani P. Galactocele in a male infant: Case report and review of literature. Pediatr Pathol. 1993;13(3):305-8.

8. Ironsides JW, Guthrie W. The galactocele: a light- and electron microscopic study. Histopathology. 1985;9(4):57-67.

9. Naveen N, Avijeet Mukherjee, Vikrant Mahajan. "A clinical study of benign breast disease in rural population". J Evol Med Dent Sci. 2013;2(30):5499-5511.

10. Ranabhat S, Subedi M, Bhandari A, et al. Clinico-pathologic profile of women with palpable breast lumps in Chitwan Medical College, Nepal. Int J Res Med Sci. 2015;3(7):1611-6.

11. Hosny IA, Salah Eldin L, Elghawabi HS. Radiological evaluation of palpable breast masses during pregnancy and lactation. Egypt J Radiol Nucl Med. 2011;42(2):267-73.

12. Adesunkanmi AR, Agbakwuru EA. Benign breast disease at Wesley Guild Hospital, Ilesha, Nigeria. West Afr J Med. 2001;20(2):146-51.

13. Adeniji KA, Adelusola KA, Odesanmi WO. Benign disease of the breast in Ile-Ife: a 10 year experience and literature review. Cent Afr J Med. 1997;43(5):140-3.

14. Anyikam A, Nzegwu MA, Ozumba BC, et al. Benign breast lesions in Eastern Nigeria. Saudi Med J. 2008;29(2):2414.

15. Tayae M, Jamor J. A rare case of giant galactocele associated with prolactinoma. Pan Afr Med J. 2017;27:97.

16. Ohene-Yeboah MO. An audit of excised breast lumps in Ghanaian women. West Afr J Med. 2005;24(3):252-5.

17. Chun YS, Taghinia A. Hyperprolactinemia and galactocele formation after augmentation mammoplasty. Ann Plast Surg. 2009;62(2):122-3.

18. Tung A, Carr N. Postaugmentation galactocele: a case report and review of literature. Ann Plast Surg. 2011;67(6):66870. 